\title{
Fatty Acid Synthase inhibition prevents palmitoylation of SARS-CoV2 Spike Protein and improves survival of mice infected with murine hepatitis virus.
}

Minhyoung Lee ${ }^{1,2^{*}}$, Michael Sugiyama ${ }^{3^{*}}$, Katrina Mekhail ${ }^{1,2^{*}}$ Elyse Latreille ${ }^{2,4}$, Negar Khosraviani $^{2}$, Kuiru Wei ${ }^{2}$, Warren L. Lee ${ }^{1,2,4,5}$, Costin Antonescu ${ }^{3}$, Gregory D. Fairn ${ }^{1,2,4,6}$

Affiliations.

${ }^{1}$ Department of Biochemistry, University of Toronto

${ }^{2}$ Keenan Research Centre, St. Michael's Hospital, Unity Health Toronto

${ }^{3}$ Department of Chemistry and Biology, Ryerson University, Toronto

${ }^{4}$ Department of Laboratory Medicine and Pathobiology, University of Toronto

${ }^{5}$ Department of Medicine, University of Toronto

${ }^{6}$ Department of Surgery, University of Toronto

*authors contributed equally to this manuscript.

Running Title: palmitoylation inhibitors attenuate CoV virulence.

Severe acute respiratory syndrome coronavirus 2 (SARS-CoV2) is the causative agent of COVID19 that has infected $>76 \mathrm{M}$ people and caused $>1.68 \mathrm{M}$ deaths. The SARSCoV2 Spike glycoprotein is responsible for the attachment and infection of target cells. The viral Spike protein serves the basis for many putative therapeutic countermeasures including vaccines, blocking and neutralizing antibodies, and decoy receptors. Here we investigated the cytosolic domain of Spike and its interaction with the protein palmitoyltransferase ZDHHC5. The Spike protein is palmitoylated on multiple juxtamembrane cysteine residues conserved among coronavirus. Increased abundance of ZDHHC5 resulted in hyper-palmitoylation, while silencing of ZDHHC5 reduced the ability of the human CoV 229E to form viral plaques in cell monolayers. Inhibition of fatty acid synthase using the pharmacological inhibitor TVB-3166 eliminated palmitoylation of SARS-CoV2 Spike. Additionally, TVB-3166 attenuated plaque formation and promoted the survival of mice from a lethal murine CoV infection. Thus, inhibition of the Spike protein palmitoylation has the potential to treat SARS-CoV-2 and other CoV infections.

\section{Introduction}

Since 2002, coronaviruses (CoV) have resulted in three zoonotic outbreaks: SARS in 2002, MERS in 2012, and SARS-CoV2 in December 2019, 2 . CoVs infect humans and animals and cause various diseases targeting different tissues, including 
respiratory, enteric, renal, and hepatic ${ }^{3,4}$. The current pandemic highlights the need for effective treatments of this family of viruses.

There are enormous efforts to develop prophylaxis and therapeutic options for SARS-CoV2, including vaccination, protease inhibitors, and soluble decoy receptors ${ }^{5-7}$. Many putative countermeasures target the Spike protein required to attach the virus to cells via binding to the angiotensin-converting enzyme (ACE2) and potentially other host proteins $^{6}$. Further, the Spike protein's proteolytic cleavage generates an S2 fragment capable of stimulating viral fusion and payload delivery into the cytosol ${ }^{8}$. However, the high mutation rates of positive-sense $(+)$ sense RNA viruses may result in resistance to some of these putative therapeutics ${ }^{9}, 10$. Additionally, the existence of many potential zoonotic coronavirus species in bat and civet populations necessitates exploring all available therapies ${ }^{11}, 12$. Thus, identifying a pan-CoV therapy that targets the host is desirable for current and potential zoonotic infections.

In the Spring of 2020, Krogan and colleagues reported that the protein palmitoyltransferase ZDHHC5 and its binding partner Golga7 interact with the Spike protein of SARS-CoV2 using an affinity purification/mass spectrometry approach ${ }^{13}$. The results suggest that the cytosolic tail of the Spike protein may be post-translationally palmitoylated. Consistent with this notion is that the Spike protein of the murine hepatitis virus (MHV) requires palmitoylation of its C-terminal tail to support virion production ${ }^{14}$. Specifically, preventing its palmitoylation prevented the MHV Spike protein from being incorporated into new virions ${ }^{14}$. Further, cells expressing a mutant version of Spike unable to be palmitoylated have a reduced ability to stimulate membrane fusion vis-àvis syncytium formation ${ }^{14,15}$. Finally, palmitoylation of the SARS Spike is critical for its ability to catalyze cell-cell fusion ${ }^{16}$. Together, these findings motivated us to determine if the SARS-CoV2 Spike protein is palmitoylated and investigate whether preventing palmitoylation could serve as a therapeutic strategy.

\section{Results and Discussion}

Alignment of the C-terminal domains of several human and murine CoVs reveals the prevalence of Cys residues within the 20 amino acids adjacent to the membrane (Figure $1 A)$. In the case of SARS-CoV2 Spike, half of the first 20 amino acids are Cys residues, thus providing ample sites for potential palmitoylation. To explore this possibility, we transiently transfected HEK293T cells with a plasmid encoding the SARS-CoV2 Spike tagged with a C-terminal C9 epitope ${ }^{17,18}$. Immunoblotting revealed numerous reactive bands in transfected cells but none in controls (data not shown). Consistent with previous reports ${ }^{18,19}$ we detect several Spike molecular species, including a full-length $\sim 190 \mathrm{kDa}, \mathrm{a} \sim 100 \mathrm{kDa}$ fragment consistent with the S2 fragment, and several high molecular weight bands that likely represent a mixture of detergent-resistant multimers.

Next, cells transiently transfected with Spike-C9 from SARS-CoV2 were cultured in the presence of 15-hexadecynoic acid (15-HDYA), an alkyne containing "clickable" palmitate analog, to determine if this fatty acid could be covalently attached to the Spike protein ${ }^{20}$. Spike-C9 was captured on anti-C9 agarose beads from cell lysates and 
subsequently reacted with azide-Cy5.5. As shown in Figure 1B, the Spike-C9 from cells incubated with alkynyl-palmitate and reacted with the Cy5.5-azide generated a similar banding pattern as the anti-C9 antibody (Figure1C), suggesting that full-length, S2 fragment and high-molecular weight species of the Spike protein are all palmitoylated.

Considering the SARS-CoV-2 Spike protein contains ten putative palmitoylation sites, we sought to examine the extent of palmitoylation on a per protein basis. We used an approach termed acyl-polyethylene glycol (PEG) exchange (APE) that involves substituting each palmitoyl group attached to the Spike protein for a 5-kDa PEG mass $\operatorname{tag}^{21}$. The unreacted protein (input) and the protein stripped of palmitate but not reacted with PEG (-PEG) both run at $190 \mathrm{kDa}$ (Figure 1D). In comparison, Spike-C9 with palmitoyl groups replaced with 5-kDa PEG displays five distinct bands consistent with an unmodified band up to and including four sites of palmitoylation. Given the proximity of the 10 Cys residues within a 20 amino acid stretch, it is unclear if the addition of PEG molecules could be constrained. Regardless, the results demonstrate that the Spike protein is indeed capable of being modified on numerous sites and that some individual proteins contain a minimum of four palmitoyl groups.

To investigate the role of the Spike palmitoylation we used a site-directed mutagenesis approach to mutate all ten Cys residues to Ser. Lysates of HEK293T cells expressing wild-type and Spike ${ }^{\mathrm{C}-\mathrm{S}}-\mathrm{C} 9$ were immunoblotted and revealed that the Spike ${ }^{\mathrm{C}-\mathrm{S}}-\mathrm{C} 9$ was expressed at comparable levels to the wild-type (Figure 2A). Immunofluorescence microscopy confirmed that both the wild-type Spike and Spike ${ }^{\mathrm{C}->\mathrm{S}}$ C9 localize to the plasma membrane (Figure 2B), consistent with previous reports, https://doi.org/10.1101/2020.10.12.335562. Previous studies have demonstrated that the Spike protein could catalyze syncytium formation provided the neighboring cells to express ACE2 ${ }^{6}$. To determine if the palmitoylation of the Spike protein is required for syncytium formation, we used a co-culture strategy where cells co-transfected with Spike-C9 and mCherry were lifted and re-seeded with cells expressing ACE2 and GFP. Co-cultures containing Spike but no ACE2 showed no signs of cell-cell fusion, as expected, while co-cultures with both Spike and ACE2 displayed numerous large syncytium that contains both GFP and mCherry [Figure 2C]. In contrast, HEK293T cells expressing Spike ${ }^{\mathrm{C}-\mathrm{S}}-\mathrm{C} 9$ co-cultured with ACE2 expressing cells displayed only a few instances of small $\mathrm{mCH}$ - and GFP-positive cells. The results demonstrate that the Cys residues are required for the Spike protein to facilitate syncytium formation.

The human genome encodes a family of 23 zinc finger Asp-His-His-Cys (ZDHHC) domain-containing palmitoyltransferases ${ }^{22}$. Yet, only ZDHHC5 interacts with the SARSCoV2 Spike ${ }^{13}$. We confirmed the previous results and found that Spike-C9 could transiently interact with HA-tagged ZDHHC5 (Figure 3A, B). Additionally, we found that in cells ectopically expressing $\mathrm{HA}-\mathrm{ZDHHC5}$, more of the Spike-C9 is palmitoylated based on the intensity and the appearance of a higher molecular weight band in the APE assay (Figure 3C). Next, we wanted to know if silencing ZDHHC5 could limit the spread of CoV in cell culture. To do this, we used the biosafety level 2 human CoV $229 \mathrm{E}$ as a model. Although the Spike protein of this CoV recognizes a different host protein, $\mathrm{CD}_{13}{ }^{23}$, its cytosolic tail is remarkably similar to that of SARS-CoV2 (Figure 
$1 \mathrm{~A})$. Using the APE assay, we found that the 229E Spike is palmitoylated on multiple sites. However, the exact number of palmitoylation sites is not apparent compared to SARS-CoV2 (Figure 1D). The banding pattern of the 229E Spike was less clear and often resulted in a smear. Regardless, the intensity of the PEGylated proteins is further enhanced when ZDHHC5 levels are increased, and a higher molecular weight is detectable (Figure 3D). This finding is consistent with the Spike proteins of SARS-CoV2 and likely 229E being substrates of ZDHHC5.

To investigate the role of ZDHHC5 in the generation of virulent progeny, we used two complementary cell-based assays. First, we silenced ZDHHC5 in confluent monolayers of MRC- 5 cells and assessed the ability of 229E to form plaques (Figure $3 E, F)$. We found that control monolayers produced an average of $\sim 10^{6} \mathrm{PFU} / \mathrm{ml}$, whereas cells with reduced levels of ZDHHC5 had substantially fewer plaques than siControl. From the plaque assay, it was unclear if the loss of ZDHHC5 was impacting the initial infection or the subsequent spread throughout the monolayer. To complement the plaque assay we used a liquid culture assay to investigate infection and dissemination. In this assay, cells were incubated in the presence of $229 \mathrm{E}$ virus at a low multiplicity of infection (MOI) $=0.005$ for 2 hours, followed by extensive washing and further incubation for 72 hours. Following the incubation period, cells were processed and stained with an antibody against the 229E Spike ${ }^{24}$ and an Alexa488 secondary antibody. As shown in Figure $3 \mathrm{G}, \mathrm{H} \approx 51 \%$ of the siControl cells were positive for the Spike protein. In contrast, $16 \%$ of the ZDHHC5 silenced cells were positive for the Spike protein. The results are consistent with the notion that ZDHHC5 is required for the generation of virulent progeny but not the initial infection of the MRC- 5 cells.

Based on our data, we sought to determine if ZDHHC5 is a suitable therapeutic target for the treatment of $\mathrm{CoV}$ infections. Unfortunately, no specific inhibitor of ZDHHC5 exists. However, we found that the fatty acid synthase (FASN) inhibitor cerulenin prevented the palmitoylation of ZDHHC5 substrates, NOD1 and NOD2 ${ }^{25}$. This is logical since FASN produces palmitoyl-CoA, the substrate for the ZDHHCs. However, cerulenin and its derivative $\mathrm{C} 75$ are not suitable therapeutically due to severe weight loss and off-target effects in murine models ${ }^{26-28}$. Fortunately, a series of heterocyclic FASN inhibitors have been developed by Sagimet Biosciences, formerly 3-V Biosciences (San Mateo, USA). One of these inhibitors, TVB-2640, is orally available, well-tolerated, and a highly potent FASN inhibitor in clinical trials for cancer (ClinicalTrials.gov ID: $\underline{\text { NCT03179904) }}^{29}$ and non-alcoholic fatty liver disease ${ }^{30}$. TVB2640 is not commercially available. However, a surrogate compound, TVB-3166, is commercially available and an effective FASN inhibitor in cell culture and mice ${ }^{31}$. Treating HEK293T cells expressing Spike-C9 with TVB-3166 or 2-bromopalmitate, a non-specific inhibitor of ZDHHC enzymes, attenuated the palmitoylation of the Spike protein (Figure 4A). Likewise, TVB-3166 was also able to partly reduce the palmitoylation of the 229E Spike protein (Figure 4B) and in PFU assay, treating cells with TVB-3166 after infection, attenuated 229E plaque formation by nearly $86 \%$ (Figure $4 C, D)$. 
The cellular results demonstrating that TVB-3166 attenuated Spike palmitoylation, and limited spread of the $229 \mathrm{E}$ virus motivated us to try this orally available FASN inhibitor in a mouse model. To do this, we nasally infected $\mathrm{A} / \mathrm{J}$ mice intranasally with $150,000 \mathrm{TCID}_{50}$ of MHV-S. In control mice, changes in health were noticed as early as two days post-infection; all proceeded to deteriorate as monitored by several health indicators, including decreased temperature, body weight, and activity. By day 5, all control mice were sacrificed as per animal care protocols. In contrast, mice treated daily with $30 \mathrm{mg} / \mathrm{kg}$ of TVB-3166 by oral gavage showed both prolonged survival and even recovery (Figure 4E). The results demonstrate that reducing palmitoyl-CoA levels is beneficial during CoV infection as this reduction promotes survival in mice.

We have established that the Spike protein of SARS-CoV2 is palmitoylated and that this modification supports its ability to catalyze membrane fusion. This observation is similar to findings on MHV and SARS Spike proteins ${ }^{14,16}$. The results suggest that palmitoylation of the $S 2$ fragment is critical to facilitate membrane fusion. However, palmitoylation of the full-length protein may also influence protein interactions or viral assembly. Indeed, the MHV Spike's palmitoylation is required for interactions with the M protein to incorporate the Spike protein into new virions ${ }^{14}$. Since no specific or clinically available inhibitors of ZDHHC5 are available, we used a FASN inhibitor, TVB-3166, to attenuate palmitoyl-CoA synthesis. We could not find any other ZDHHC isoforms in affinity purification - mass spectrometry experiments consistent with Krogan and colleagues. However, there is the possibility that another ZDHHC may also be able to modify the Spike protein. Additionally, the envelope (E) protein of MHV also requires palmitoylation for the assembly of virions. Thus, reducing palmitoyl-CoA levels may prevent the palmitoylation of multiple CoV proteins. Treating cells or mice with TVB3166 limited the ability of human CoV 229E to produce virulent progeny in cell culture and promoted survival of mice subjected to a lethal MHV infection. We suggest that our findings warrant further COVID studies using the structurally related TVB-2640 that has already advanced to Phase II clinical trials for fatty liver disease and cancer ${ }^{30,32}$.

Alterations in lipid metabolism are documented in CoV infections. Sera from patients with COVID19 have altered apolipoproteins and lipid levels ${ }^{33}$, while 229E infected cells have been shown to have increased levels of free fatty acids ${ }^{33}$. As enveloped viruses, CoVs-induced remodeling of the cellular lipidome may support viral replication. Additionally, the ectodomain of SARS-CoV2 Spike contains a binding pocket for another fatty acid, linoleic acid ${ }^{34}$. The importance of this pocket is unclear, but it is conserved in other pathogenic CoVs. Further studies on the importance of fatty acids and lipid metabolism are needed to clarify these aspects of CoV infections.

We anticipate that our findings will advance the possibility of using TVB-2640 or other FASN inhibitors to treat COVID-19 or other CoV infections. Since FASN is a host enzyme, the likelihood of viral resistance is low and would necessitate extensive mutagenesis of the Spike protein to bypass the requirement for palmitoylation. Clinically, the identification and approval of an orally available pan-CoV treatment or prophylaxis would be beneficial in the short-term and the future against other zoonotic CoVs. 


\section{Acknowledgments}

This work was supported by the St. Michael's Hospital Foundation and a Project Grant from the Canadian Institutes of Health Research (PJT166010) to GDF. WLL is supported by a Canada Research Chair in Mechanisms of Endothelial Permeability and operating funds from the Keenan Foundation and a CHRP grant (CPG 158284; CHRP 523598) from the CIHR/NSERC. CA received funding from the Ryerson Faculty of Science and the Ryerson COVID-19 SRC Response Fund. ML is supported by a doctoral scholarship from the Natural Sciences and Engineering Research Council (NSERC) of Canada. EL is supported by a Canadian Graduate Scholarship - Master's Program and a Scholarship from the St. Michael's Hospital Research Training Center Scholarship.

\section{Disclosures}

The patent to TVB-2640 and related compounds belong to Sagimet Biosciences (San Mateo, California). The authors receive no financial compensation or support from Sagimet Biosciences. The authors do not hold stock or interest in Sagimet Biosciences.

\section{Figure Legends}

Fig. 1. Palmitoylation of SARS-CoV-2 Spike protein. (A) Sequence alignment of the cytosolic amino acids adjacent to the transmembrane domain of Spike proteins of various human and murine coronaviruses. Adapted from Clustal $\Omega ;\left(^{*}\right)$ residue is wholly conserved, (:) strongly similar properties, (.) weakly similar properties conserved. The highlighted cysteine residues were previous shown to be palmitoylated (B) Metabolic labeling of alkynyl-palmitate (15-HDYA) on SARS-CoV-2 Spike-C9 ectopically in HEK293T cells. Covalently attached alkynyl palmitate was reacted with Cy5.5-azide using $\mathrm{Cu}^{+2}$-catalyzed click chemistry. Protein samples separated by SDS-PAGE were subjected to in-gel fluorescence signal detection on a LI-COR Odyssey. (C) Immunoblot of SARS-CoV-2 Spike-C9 shown in B. (D) Acyl-PEG exchange assay (APE) of SARSCoV-2 Spike-C9 in HEK293T cells. Total lysates were subjected to APE and analyzed by immunoblotting with an anti-C9 antibody. Input: unprocessed HEK293T lysate. +PEG: de-palmitoylated and PEG added. -PEG: de-palmitoylated without PEG. (E) Magnified section of pegylated Spike with low contrast (left) and high contrast (right). Magenta arrowheads indicate individual bands.

Fig. 2. Palmitoylation of Spike is required for cell-cell fusion. (A) Western blot of SARS-CoV-2 Spike-C9 of the ten Cys to Ser mutant. (B) Immunofluorescence of SARS-CoV-2 Spike and the Spike ${ }^{\mathrm{C}>\mathrm{S}}-\mathrm{C} 9$ mutant. Scale bars, $10 \mu \mathrm{m}$ (C) Co-culture of HEK cells expressing Spike-C9 and ACE2 form syncytium. HEK293T cells cotransfected with mCherry-C1 (magenta), and SARS-CoV-2 Spike-C9 (left and mid) or SARS-CoV-2 Spike ${ }^{\mathrm{C}-\mathrm{S}}-\mathrm{C}$-9 (right) were co-cultured with cells transfected with EGFP-C1 
(left, cyan) or and myc-ACE2 (mid, right). Scale bars, $20 \mu \mathrm{m}$. Arrowheads indicate fused cells.

Fig. 3. ZDHHC5 palmitoylates Spike proteins and is required for viral plaque formation. (A, B) Co-immunoprecipitation of SARS-CoV-2 Spike-C9 with 3xHAZDHHC5. HEK293T cells co-transfected with Spike-C9 and 3xHA-ZDHHC5 were immunocaptured and probed as indicated. (C, D) Increased expression of ZDHHC5 increases the levels of Spike palmitoylation. The APE assay of SARS-CoV-2 Spike (C) and Human CoV 229E (D) in HEK293T cells co-expressing 3xHA-ZDHHC5. (E) Viral PFU assay of MRC-5 cells infected with 229E and stained with crystal violet. (F) quantification of plaque assays infected with different stocks of 229E virus (L2 and L5). Unpaired t-test, ${ }^{* *}=\mathrm{p}<0.005$. (G) Liquid viral infection assay of MRC-5 cells infected with an $\mathrm{MOI}$ of 0.0005 of $229 \mathrm{E}$ virus, stained with $229 \mathrm{E}$ Spike neutralizing antibody ${ }^{35}$ and Alexa488 conjugated secondary antibody. Secondary antibody pseudocolored in cyan and DAPI in yellow. Acquired at a 10x magnification, scale bars, 50 $\mu \mathrm{m}$. $(\mathbf{H})$ Quantification of liquid viral infection assay where each data point represents a 10x image field where and average of 600 cells were counted in imageJ. Unpaired t-test, $* * * *=p<0.0001$.

Fig. 4. Pharmacological inhibition of Fatty Acid Synthase blocks Spike Palmitoylation and limits viral infection. (A) APE assay of SARS-CoV-2 Spike in HEK293T treated with TVB-3166 or 2-Bromohexadecanoic acid (2-BP). Cells were transfected with CoV-2 Spike-C9 and treated with TVB-3166 $(0.2 \mu \mathrm{M}$ or $20 \mu \mathrm{M})$ and 2-BP $(50 \mu \mathrm{M})$ overnight. Samples were processed and proteins visualized by immunoblotting.

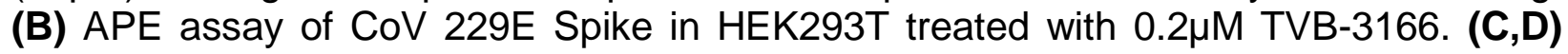
Human CoV 229E-mediated plaque assay in MRC-5 cells in the presence of TVB-3166. MRC-5 cells were infected with $3.34 \times 10^{5} \mathrm{PFU} / \mathrm{mL}$ of Human CoV 229E for four days with and without $0.2 \mu \mathrm{M}$ TVB-3166, Unpaired t-test, $\mathrm{p}<0.0001$.(E) Survival of mice challenged by a lethal dose of MHV-S. ${ }^{* * *}=p<0.005$.

\section{Materials and Methods}

Reagents: TVB-3166 (SML1694), 2-Bromohexadecanoic acid (238422), EDTA-free protease inhibitor (1183617001), TCEP (C4706), tert-butanol (471712) were purchased from Millipore-Sigma (Oakville, Canada). Phusion polymerase, Xhol and Nhel FastDigest restriction enzymes, T4 DNA ligase, dithiobissuccinimidyl propionate (DSP), Protein G Sepharose, Lipofectamine 3000, RNAiMAX, Eagle's Minimum Essential Medium (EMEM), SuperSignal West Pico were purchased from Thermo Fisher Scientific (Mississauga, Canada). Dulbecco's modified Eagle's medium (DMEM), Temin's Modified Eagle Medium (11935046), fetal bovine serum (FBS), charcoal stripped FBS and Trypsin/EDTA were obtained from Wisent Bio Products (St. Bruno, Canada). Triton-X100, DMSO and $\mathrm{CuSO}_{4}$ were from BioShop Canada Inc (Burlington, Canada), alkynyl-palmitate (Click Chemistry Tools; Scottsdale, AZ), Cy5.5-azide 
(Lumiprobe; Hunt Valley, Maryland), Site Counter (Badrilla' Leeds, UK), PFA (Electron Microscope Science; Hatfield, PA),

Plasmids: pcDNA3.1-SARS2-Spike (Addgene plasmid \#145032), pCEP4-myc-ACE2 (Addgene plasmid \#141185). PBcmv-229E-wt_WRPE ${ }^{24}$ (from Jim Rini, University of Toronto), HA-tagged ZDHHC5 ${ }^{25}$, EGFP-C1 (Clontech), mCherry-C1 (Clontech). The 229E Spike CDNA was amplified by PCR with the forward primer: 5'TTTTTTGCGGCTAGCATGTTCGTGCTGCTGG and the reverse primer: 5'TTTTTTGCGCTCGAGTCACGCCGGCGCCACCTGGCTGGTTTCGGTCTGGATGTGG ATCTTTTCCA using Phusion polymerase (New England Biolabs) according to manufacturer's instruction. The amplified 229E sequence and SARS-CoV2 S plasmid were then incubated with Xhol and Nhel restriction enzymes at $37^{\circ} \mathrm{C}$ for 1 hour. The reactions were then heat inactivated and purified with a PCR clean-up kit. The insert and vector were then incubated with T4 DNA ligase at $16^{\circ} \mathrm{C}$ overnight. The ligation mixture was transformed into NEB 5-alpha high efficiency $E$. Coli and plated on LB plates with the appropriate antibiotic. Plasmids were then harvested with a midi prep kit following the manufacturer's instructions.

Mutagenesis of SARS CoV-2 Spike multi-cysteine to serine: The SARS-CoV-2 Spike protein has 10 cysteines in its cytosolic tail; C1235, C1236, C1240, C1241, C1243, C1247, C1248, C1250, C1253, C1254. In this study, all 10 Cys residues were replaced by Ser. Sequential PCR and overlap extension PCR was used for the complete substitution strategy. Using pcDNA3.1 SARS CoV-2 Spike-C9 as an initial template, two fragments were generated: 5'-, and 3'-terminal fragments. To amplify 3'terminal fragment (contains 10 Cys to Ser mutation), three sequential PCR reactions were performed by using overlapping forward primers (F2 primer overlaps with F1 primers, F3 primer overlaps with F2 primers). As a result, fragment 1 served as a DNA template of fragment 2, and fragment 2 as a template of fragment 3 . The fragment 1,2, and 3 were generated using forward primers $\mathrm{F} 1$ - 5'CTCCTCCTCCTCCGGCAGCTCCTCCAAGTTCGATGAGGACGATAG-3', F2- 5'CCTCCTCCTCCAGCTCCCTGAAGGGCTCCTCCTCCTCCGGCAGCT-3', and F3- 5TGATGGTGACCATCATGCTGTCCTCCATGACCTCCTCCTCCAGCTCCCTG-3',

respectively, and reverse primer 5'- TCTAGACTCGAGCTAAGCGGGAGC-3'. To amplify 5' DNA fragment, forward primer 5'- CAAGCTGGCTAGCATGTTTGTCTTCC-3' and reverse primer 5'-TCATGGAGGACAGCATGATGGTCACCATCA-3' were used. The 5' DNA fragment and 3'DNA fragment were annealed together with their complementary overhanging by PCR using the primer pairs 5'CAAGCTGGCTAGCATGTTTGTCTTCC-3' and 5'TCTAGACTCGAGCTAAGCGGGAGC-3' to generate full length DNA. All PCR thermocycle conditions were based on Touchdown $\mathrm{PCR}^{36}$. The multi-site mutated SARS CoV-2 Spike was then subcloned into the pcDNA3.1 vector using Nhel and Xhol restriction digestion.

Cell culture: HEK293T cells were cultured in DMEM supplemented with $10 \%$ FBS at $37^{\circ} \mathrm{C}$ and $5 \mathrm{CO}_{2}$. MRC- 5 cells (ATCC) were cultured in EMEM supplemented with $10 \%$ FBS and 1\% Penicillin/Streptomycin (Gibco) 
Syncytium formation assay: HEK293T cells were seeded in 6-well plates and transfected with: 1. EGFP-C1 vector with myc-ACE2 vector and 2. mCherry-C1 vector with CoV-2 Spike-C9 or Spike multi-cysteine to serine mutant. After 16-24 hrs of incubation, cells were lifted by trypsinization and co-cultured overnight. Fluorescent images were acquired through EVOS FLoid ${ }^{\mathrm{TM}}$ Cell Imaging System.

Antibodies and antibody-conjugated beads: C9 antibody (Clone 1D4, Santa Cruz, sc-57432), anti-C9 agarose bead (Cube Biotech), HA antibody (Abcam, ab9110), myc antibody (Bio X Cell, Lebanon, NH), SARS-CoV-2 Spike antibody, (Abcam, ab272504), ZDHHC5 antibody (Sigma, HPA014670), fluorescent secondary antibodies (Jackson Laboratories, Invitrogen, LI-COR).

siRNA Transfection: MRC-5 cells seeded on 6-well tissue culture plates were transfected 2X, 24 hours apart, followed by a 24 to 48-hour recovery before infection with $229 \mathrm{E}$. For transfection, cells were initially transfected at $50 \%$ confluence in $1 \mathrm{~mL}$ Opti-MEM Reduced-Serum Medium (Gibco). A siRNA transfection master mix of Lipofectamine RNAiMAX, siRNA (CTRL or DHHC5), and Opti-MEM was made according to manufacturer's instructions, with a final siRNA concentration of $50 \mathrm{nM}$. Cells were transfected with the siRNA master mix for 4 hours, followed by a change to growth media. After the final transfection, media was changed to growth media and cells were given 24 to 48-hour recovery before the infection, at which point they were at $100 \%$ confluence. Oligo sequences used CTRL non-targeting siRNA: CGUACUGCUUGCGAUACGGUU and ZDHHC5 siRNA: CUGUGAAGAUCAUGGAUAAUU [PMID: 317807750]

Immunoblotting: SDS polyacrylamide gels were transferred on PVDF membranes by Trans-blot Turbo System at 25 Volts for 20 min. Membranes were blocked with 5\% BSA for 1 hour at room temperature and were incubated with primary antibodies diluted in blocking solution at $4^{\circ} \mathrm{C}$ overnight. Membranes were washed with TBS- $0.1 \%$ Tween 20 (TBST) for 5 minutes, 3 times and were subsequently incubated with appropriate secondary antibodies. Blots were then washed three times with TBST for 5 minutes each wash. The membranes were treated with ECL and imaged with ChemiDoc Imaging System (BioRad).

Co-Immunoprecipitation: HEK293T cells seeded in T25 Flask $\left(25 \mathrm{~cm}^{2}\right)$ were grown until reaching $70-90 \%$ confluency. Cells were transfected with $3 \times \mathrm{HA}-\mathrm{zDHHC5}$ and CoV2 Spike-C9 plasmids using Lipofectamine 3000 according to the manufacturer's instruction and incubated for $16-24 \mathrm{hrs}$. For protein cross-linking, cells were incubated with $2 \mathrm{mM}$ DSP crosslinker in PBS at $4^{\circ} \mathrm{C}$ for $2 \mathrm{hrs}$. DSP was quenched in $20 \mathrm{mM}$ glycine in PBS for $15 \mathrm{~min}$, and cells were lysed in NP40 lysis buffer (20mM Tris and adjust to $\mathrm{pH} 7.4,150 \mathrm{mM} \mathrm{NaCl}, 1 \% \mathrm{NP} 40,2 \mathrm{mM}$ EDTA) supplemented with cOmplete Protease Inhibitor Cocktail tablet, EDTA-Free at a constant agitation for $30 \mathrm{~min}$ at $4^{\circ} \mathrm{C}$. Cell lysates were centrifuged at $12,000 \times \mathrm{xg}$ for $20 \mathrm{~min}$, and supernatant was collected. Samples were pre-cleared by incubating with protein $G$ Sepharose beads and were spun at $14,000 \mathrm{~g}$ for $1 \mathrm{~min}$ to collect the supernatant. Pre-cleared samples were incubated with anti-C9 or anti-HA primary antibody for overnight at $4^{\circ} \mathrm{C}$. To form primary antibody-bead complex, Protein $\mathrm{G}$ beads were added and incubated for $30 \mathrm{~min}$. Beads 
were washed in cold wash buffer (20mM Tris, pH 7.4, $350 \mathrm{mM} \mathrm{NaCl}, 1 \%$ NP-40, $2 \mathrm{mM}$ EDTA) 6 times, and proteins were eluted with SDS Laemmli buffer and heated for $30 \mathrm{~min}$ at $37^{\circ} \mathrm{C}$ prior to SDS-PAGE.

Immunofluorescence: Cells were seeded on a 18mm-round coverslip in 12-well plate. Cells expressing myc-ACE2, CoV-2 Spike or CoV-2 Spike ${ }^{\text {- }>S}-C 9$ were fixed with $4 \%$ PFA. Remaining PFA was quenched by $0.15 \mathrm{M}$ glycine, and cells were permeabilized by $0.1 \%$ Triton-X100 for 20 min at RT. Cells were blocked with $5 \%$ BSA in PBS for 1 hr and probed with a primary antibody (human myc, CoV-2 Spike) in 5\% BSA for $1 \mathrm{hr}$. Cells were washed with PBS and probed with fluorescently labelled secondary antibodies for $1 \mathrm{hr}$.

Confocal Microscopy: Imaging in the St. Michael's Hospital Biolmaging was conducted using an Andor Diskcovery multi-modal imaging system provided by Quorum Technology (Guelph, Ontario). The system is based on a Leica DMi8 equipped with a 63x/1.47 NA oil objective; 405, 488, 561, and 637 nm laser lines; 450/50, 525/50, $600 / 50,610 / 75$, and 700/75 emission filters. Images were acquired using Metamorph software on a Hamamatsu ORCA-Flash 4.0 V2 sCMOS camera. Imaging at Ryerson University was performed on the Quorum WaveFX spinning disc based on an inverted Leica DMi8 equip with an Andor Zyla 4.2 sCMOS camera using a 10x objective controlled by MetaMorph.

Metabolic labelling/ on-bead click chemistry assay: HEK293T cells seeded in T25 Flask $\left(25 \mathrm{~cm}^{2}\right)$ were transfected with pcDNA3.1-SARS CoV-2- Spike-C9 and incubated overnight in DMEM supplemented with $10 \%$ (v/v) charcoal-stripped FBS with or without $100 \mu \mathrm{M}$ 15-HPYA (Click Chemistry Tools, Scottsdale, Arizona). After 18-24 hours of labeling, cells were washed twice with ice-cold D-PBS and collected following scraping. Cells were lysed in IP buffer (20mM Tris and adjust to $\mathrm{pH} 8.0,137 \mathrm{mM} \mathrm{NaCl}, 1 \% \mathrm{NP} 40$, 2 mM EDTA) supplemented with cOmplete Protease Inhibitor Cocktail tablet, EDTAFree (Roche) and Immunoprecipitated using C9-agarose bead (Cube biotech) at $4^{\circ} \mathrm{C}$ overnight. The beads were then washed with ice-cold D-PBS 4 times and incubated in D-PBS supplemented with $1 \mu \mathrm{M}$ Cy5.5-azide (Click Chemistry Tools), $1 \mathrm{mM}$ TCEP (adjusted to $\mathrm{pH} 7.0$ ), $1 \mathrm{mM} \mathrm{CuSO}$, $0.1 \mathrm{mM}$ TBTA (1:4 v/v DMSO: tert-Butanol) for $2 \mathrm{~h}$. The beads were washed 5 times with D-PBS, and proteins were eluted with 5X SDS Laemmli buffer. Protein samples were resolved by SDS-PAGE and subjected to in-gel fluorescence using the $685 \mathrm{~nm}$ laser on the Li-Cor Odyssey Infrared Imaging System.

Acyl-PEG exchange assay: HEK293T cells were seeded in 6 well plates in DMEM $+10 \%$ FBS and were transfected with lipofectamine 3000 reagent. For each well $2.5 \mathrm{mg}$ of either: 229E Spike-C9 + HA-ZDHHC5, 229E Spike-C9+ EGFP-C1, SARS-CoV-2 Spike-C9 and HA-ZDHHC5 or SARS-CoV2 Spike-C9 + EGFP-C1 DNA mixtures were diluted in Opti-mem and mixed with Lipofectamine 3000 according to the manufacturer's instructions. Upon transfection, cells were treated with the indicated concentrations of TVB-3166, or $50 \mu \mathrm{M}$ 2-BP. 16-18 hours post transfection cells were lysed and processed with the SiteCounter ${ }^{\mathrm{TM}}$ S-palmitoylated protein kit (Badrilla, Leeds, UK) 
following the manufacturer instructions. Samples were subjected to SDS-PAGE by resolving on a $3-8 \%$ Criterion $^{\mathrm{TM}}$ XT Tris-Acetate gel (Bio-Rad) for immunoblotting.

Propagation of the Human 229E Coronavirus: Human coronavirus 229E (ATCC ${ }^{\circledR}$ VR$740^{\mathrm{TM}}$ ) was purchased from American Type Culture Collection (ATCC), Manassas, VA. Stocks of the $229 \mathrm{E}$ virus were produced by propagation in MRC-5 cells. Briefly, a T75 flask of $90 \%$ confluent MRC- 5 cells were infected with a MOI of 0.01 for 2 hours at $33^{\circ} \mathrm{C}$ in infection media for viral adsorption. Unbound virus was removed by washing cells $2 X$ with infection media, and infected cells were returned to the $33^{\circ} \mathrm{C}$ incubator in $12 \mathrm{~mL}$ infection media for 72 hours. The virus-containing supernatant was centrifuged at 1000 $\mathrm{x} \mathrm{g}$ for 5 minutes to remove cellular debris, and single use aliquots of the virus were frozen at $-80^{\circ} \mathrm{C}$.

Human CoV 229E plaque forming unit assay: PFU assays was used to determine viral titers and experiments using protocols adapted from previous publications ${ }^{37,}{ }^{38}$. Briefly, MRC-5 cells seeded on 6 -well tissue culture and grown to $100 \%$ confluency in EMEM+ 10\% FBS+ 1\% PenStrep. Plates were infected with serially diluted virus in infection media (EMEM+2\% FBS $+1 \%$ PenStrep) for 1 hour at $33^{\circ} \mathrm{C}$ in a volume of $300 u L$ with periodic agitation. After virus adsorption, unbound virus was removed by washing with infection media followed by agarose overlay with 1X MEM Temin's modification (Thermo Fisher, 11935046) $+0.3 \%$ agarose. Cells were then returned to the $33^{\circ} \mathrm{C}$ incubator until they showed obvious signs of cytopathic effects (typically 4-7 days post-infection), upon which plates were fixed with $10 \%$ neutral-buffered formalin for 4 hours, followed by removal of the agarose plug and staining with $1 \%$ crystal violet for 15 minutes. Plaques were counted based on standard protocols for determining viral titer.

Liquid viral infection assay: MRC-5 cells were seeded on glass coverslips and transfected as described above. Cells were infected with $229 \mathrm{E}$ at an $\mathrm{MOI}$ of 0.005 for 2 hours in a total volume of $2 \mathrm{~mL}$ per well. After virus absorption, cells were washed $2 \mathrm{X}$ with infection media and then replaced with $2 \mathrm{~mL}$ infection for incubation at $33^{\circ} \mathrm{C}$. After 72 hours, cells were fixed with $4 \%$ PFA for 1 hour, followed by: $0.15 \%$ glycine, $0.1 \%$ Triton X-100, 3\% BSA, for 15 minutes each. Cells were then incubated in a 1:50 dilution of mouse anti-229E Spike protein antibody by inverted drop for 1 hour at room temperature. After washing, cells were incubated in 1:1000 anti-mouse Alexa Fluor 488 secondary antibody (Jackson ImmunoResearch) and DAPI (1 mg/mL) for $1 \mathrm{~h}$ at room temperature, followed by mounting on slides with DAKO mounting media. Images were acquired with the WaveFX system. Total fluorescent signal was quantified with ImageJ.

MHV-S infection of mice: Four-week-old female $A / J$ mice were purchased from Jackson Laboratories. Mice were housed in the St. Michael's Hospital Vivarium on a standard light:dark cycle and were given free access to food and water. Prior to infection, mice were acclimatized for 1 week. Mouse infection experiments were performed in the dedicated BSL-2 room in the vivarium and procedures were conducted in accordance with the St. Michael's Hospital Animal Care Committee guidelines for animal use, under an approved animal protocol (ACC962, St. Michael's Hospital). One day prior to infection, the necks of mice were shaved and chemical depilatory cream 
was used to remove all hair in order to obtain optimal oxygen saturation measurements during the study. For infection, mice were sedated with $5 \%$ isoflurane and infected intranasally with 150,000 TCID50/mouse of MHV-S (Cedarlane, VR-766), which is known to induce pneumonitis (PMID: 17041219). The virus was diluted in PBS to a final volume of $75 \mu \mathrm{L}$ prior to administration. After infection, mice were allowed to recover for ten minutes in a cage on a covered heating pad. On the day of infection, TVB-3166 (Sigma, SML 1694) was reconstituted by adding a small volume of DMSO (10\% of the final volume) to the vial. The vial was briefly vortexed and heated in a $37^{\circ} \mathrm{C}$ water bath, then corn oil was added to make a $5 \mathrm{mg} / \mathrm{mL}$ solution for treatments. Following recovery, mice were randomly separated into weight matched groups and immediately received solvent control or TVB-3166 $(30 \mathrm{mg} / \mathrm{kg})$ by oral gavage. For the remainder of the study, mice were monitored daily and received one dose of TVB-3166 or solvent control per day. As previously reported (PMID: 26046800), mice were sacrificed if they reached two of four of the following endpoints: weight loss of $30 \%$ of the initial weight, body temperature below $31^{\circ} \mathrm{C}$, oxygen saturation below $75 \%$ or activity level of 1 (moribund). For the measurement of oxygen saturation on awake mice, the Mouse Ox Plus device and software from Starr Life Sciences was used. Accurate readings were obtained until day 3 when regrowth of hair and weight loss prevented reliable measurements.

\section{References}

1. Wang, C., Horby, P.W., Hayden, F.G. \& Gao, G.F. A novel coronavirus outbreak of global health concern. Lancet 395, 470-473 (2020).

2. da Costa, V.G., Moreli, M.L. \& Saivish, M.V. The emergence of SARS, MERS and novel SARS-2 coronaviruses in the 21st century. Arch Virol 165, 1517-1526 (2020).

3. To, K.F. et al. Tissue and cellular tropism of the coronavirus associated with severe acute respiratory syndrome: an in-situ hybridization study of fatal cases. J Pathol 202, 157-163 (2004).

4. Weiss, S.R. \& Leibowitz, J.L. Coronavirus pathogenesis. Adv Virus Res 81, 85-164 (2011).

5. Monteil, V. et al. Inhibition of SARS-CoV-2 Infections in Engineered Human Tissues Using Clinical-Grade Soluble Human ACE2. Cell 181, 905-913 e907 (2020).

6. Hoffmann, M. et al. SARS-CoV-2 Cell Entry Depends on ACE2 and TMPRSS2 and Is Blocked by a Clinically Proven Protease Inhibitor. Cell 181, 271-280 e278 (2020).

7. Kim, Y.C., Dema, B. \& Reyes-Sandoval, A. COVID-19 vaccines: breaking record times to first-in-human trials. NPJ Vaccines 5, 34 (2020).

8. Hoffmann, M., Kleine-Weber, H. \& PohImann, S. A Multibasic Cleavage Site in the Spike Protein of SARS-CoV-2 Is Essential for Infection of Human Lung Cells. Mol Cell 78, 779784 e775 (2020).

9. Duffy, S., Shackelton, L.A. \& Holmes, E.C. Rates of evolutionary change in viruses: patterns and determinants. Nat Rev Genet 9, 267-276 (2008).

10. Duffy, S. Why are RNA virus mutation rates so damn high? PLoS Biol 16, e3000003 (2018).

11. Cui, J., Li, F. \& Shi, Z.L. Origin and evolution of pathogenic coronaviruses. Nat Rev Microbiol 17, 181-192 (2019).

12. Liu, L. et al. Natural mutations in the receptor binding domain of spike glycoprotein determine the reactivity of cross-neutralization between palm civet coronavirus and severe acute respiratory syndrome coronavirus. J Virol 81, 4694-4700 (2007). 
13. Gordon, D.E. et al. A SARS-CoV-2 protein interaction map reveals targets for drug repurposing. Nature 583, 459-468 (2020).

14. Thorp, E.B., Boscarino, J.A., Logan, H.L., Goletz, J.T. \& Gallagher, T.M. Palmitoylations on murine coronavirus spike proteins are essential for virion assembly and infectivity. $J$ Virol 80, 1280-1289 (2006).

15. Shulla, A. \& Gallagher, T. Role of spike protein endodomains in regulating coronavirus entry. J Biol Chem 284, 32725-32734 (2009).

16. Petit, C.M. et al. Palmitoylation of the cysteine-rich endodomain of the SARScoronavirus spike glycoprotein is important for spike-mediated cell fusion. Virology 360 , 264-274 (2007).

17. Molday, L.L. \& Molday, R.S. 1D4: a versatile epitope tag for the purification and characterization of expressed membrane and soluble proteins. Methods Mol Biol 1177, 1-15 (2014).

18. Shang, J. et al. Structural basis of receptor recognition by SARS-CoV-2. Nature 581, 221-224 (2020).

19. Ou, X. et al. Characterization of spike glycoprotein of SARS-CoV-2 on virus entry and its immune cross-reactivity with SARS-CoV. Nat Commun 11, 1620 (2020).

20. Gaebler, A. et al. Alkyne lipids as substrates for click chemistry-based in vitro enzymatic assays. J Lipid Res 54, 2282-2290 (2013).

21. Percher, A. et al. Mass-tag labeling reveals site-specific and endogenous levels of protein S-fatty acylation. Proc Natl Acad Sci U S A 113, $4302-4307$ (2016).

22. Salaun, C., Locatelli, C., Zmuda, F., Cabrera Gonzalez, J. \& Chamberlain, L.H. Accessory proteins of the zDHHC family of S-acylation enzymes. J Cell Sci 133 (2020).

23. Nomura, R. et al. Human coronavirus 229E binds to CD13 in rafts and enters the cell through caveolae. $J$ Virol 78, 8701-8708 (2004).

24. Li, Z. et al. The human coronavirus HCoV-229E S-protein structure and receptor binding. Elife 8 (2019).

25. Lu, Y. et al. Palmitoylation of NOD1 and NOD2 is required for bacterial sensing. Science 366, 460-467 (2019).

26. Loftus, T.M. et al. Reduced food intake and body weight in mice treated with fatty acid synthase inhibitors. Science 288, 2379-2381 (2000).

27. Montesdeoca, N., Lopez, M., Ariza, X., Herrero, L. \& Makowski, K. Inhibitors of lipogenic enzymes as a potential therapy against cancer. FASEB J 34, 11355-11381 (2020).

28. Flavin, R., Peluso, S., Nguyen, P.L. \& Loda, M. Fatty acid synthase as a potential therapeutic target in cancer. Future Oncol 6, 551-562 (2010).

29. Singh, S., Karthikeyan, C. \& Moorthy, N. Recent Advances in the Development of Fatty Acid Synthase Inhibitors as Anticancer Agents. Mini Rev Med Chem 20, 1820-1837 (2020).

30. Syed-Abdul, M.M. et al. Fatty Acid Synthase Inhibitor TVB-2640 Reduces Hepatic de Novo Lipogenesis in Males With Metabolic Abnormalities. Hepatology 72, 103-118 (2020).

31. Heuer, T.S. et al. FASN Inhibition and Taxane Treatment Combine to Enhance Antitumor Efficacy in Diverse Xenograft Tumor Models through Disruption of Tubulin Palmitoylation and Microtubule Organization and FASN Inhibition-Mediated Effects on Oncogenic Signaling and Gene Expression. EBioMedicine 16, 51-62 (2017).

32. Jones, S.F. \& Infante, J.R. Molecular Pathways: Fatty Acid Synthase. Clin Cancer Res 21, 5434-5438 (2015).

33. Shen, B. et al. Proteomic and Metabolomic Characterization of COVID-19 Patient Sera. Cell 182, 59-72 e15 (2020).

34. Toelzer, C. et al. Free fatty acid binding pocket in the locked structure of SARS-CoV-2 spike protein. Science 370, 725-730 (2020). 
35. Wong, A.H.M. et al. Receptor-binding loops in alphacoronavirus adaptation and evolution. Nat Commun 8, 1735 (2017).

36. Korbie, D.J. \& Mattick, J.S. Touchdown PCR for increased specificity and sensitivity in PCR amplification. Nat Protoc 3, 1452-1456 (2008).

37. Milewska, A. et al. HTCC: Broad Range Inhibitor of Coronavirus Entry. PLoS One 11, e0156552 (2016).

38. Baer, A. \& Kehn-Hall, K. Viral concentration determination through plaque assays: using traditional and novel overlay systems. J Vis Exp, e52065 (2014). 


\section{Figure 1.}

bioRxiv preprint doi: https://doi.org/10.1101/2020.12.20.423603; this version posted December 21, 2020. The copyright holder for this preprint

A

229E SARS SARS-Cov2 MHV-1 MHV-S MERS

B

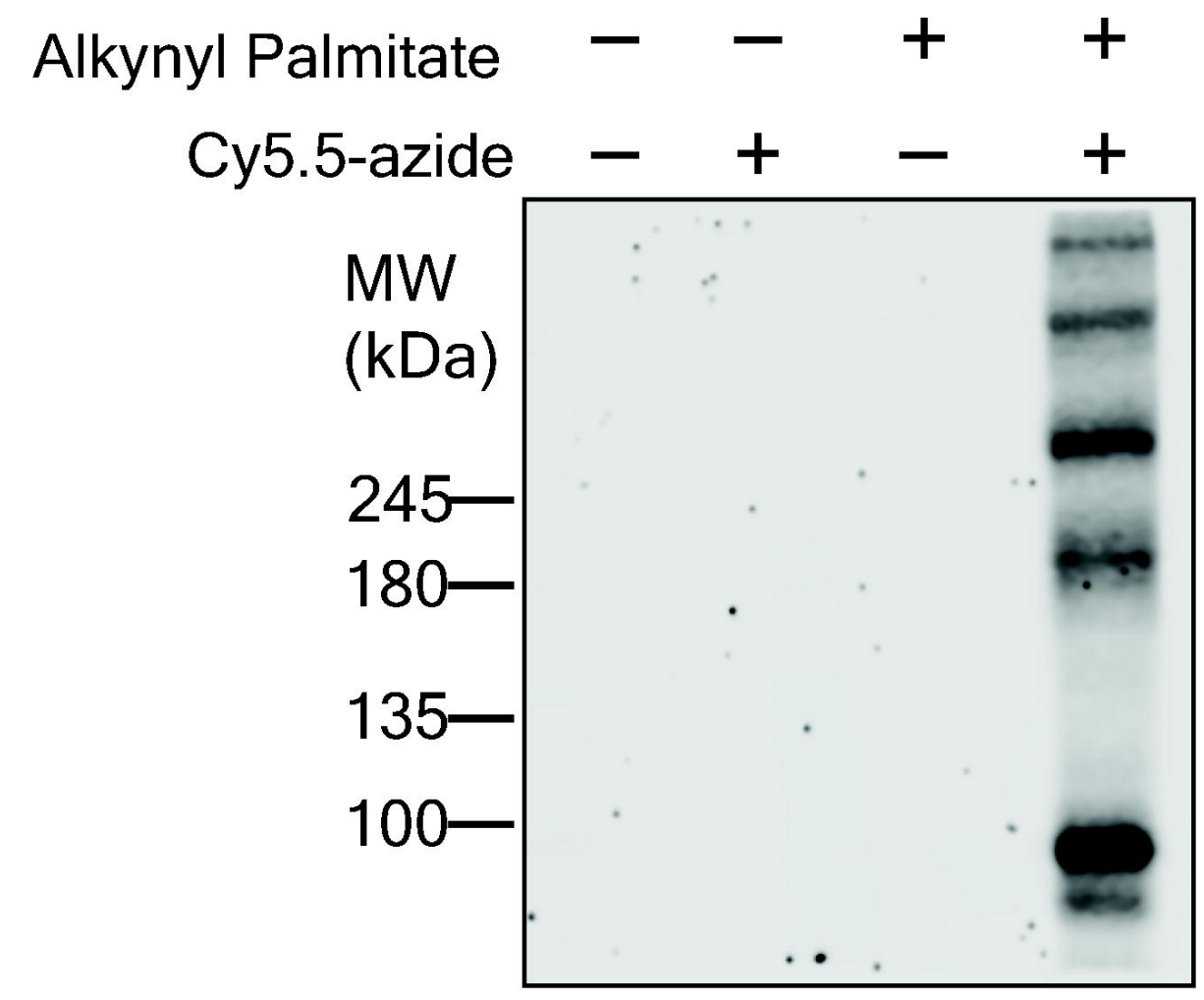

D

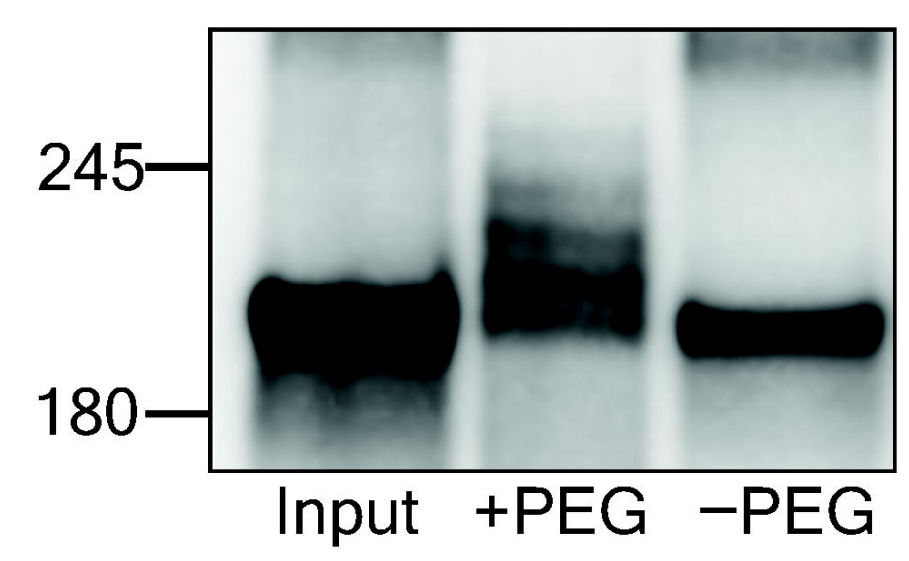

1136 CCCSTGCCGFFSCFASS IRGCCESTKLPYYDVE---KIHIQ- - 1173 1217 CCM-TSCCSCLKG-ACSCGSCCK-FD--EDDSEPVLKGVKLHYT 1255 1235 CCM-TSCCSCLKG-CCSCGSCCK-FD-EDDSEPVLKGVKLHYT 1273 1326 CCC-TGCGSCCEK---KCGNCCDEYG-GH@DS IVIHNISSHED 1363 1324 CCC-TGCGSCCFK---KCGNCCDEYG-GH@DS IVIHNISSHED 1361

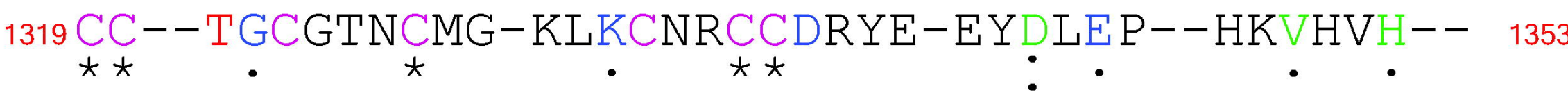

C

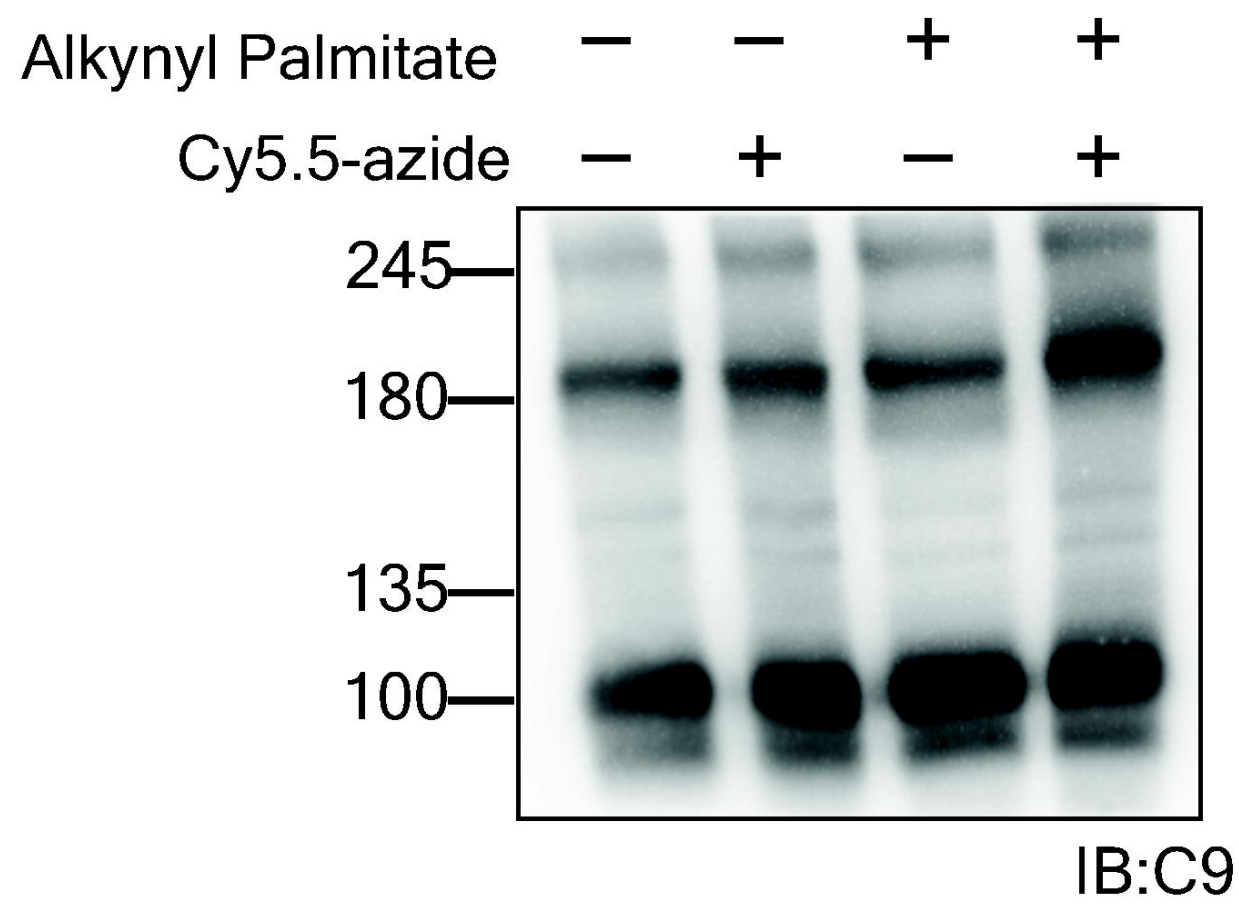

$E$

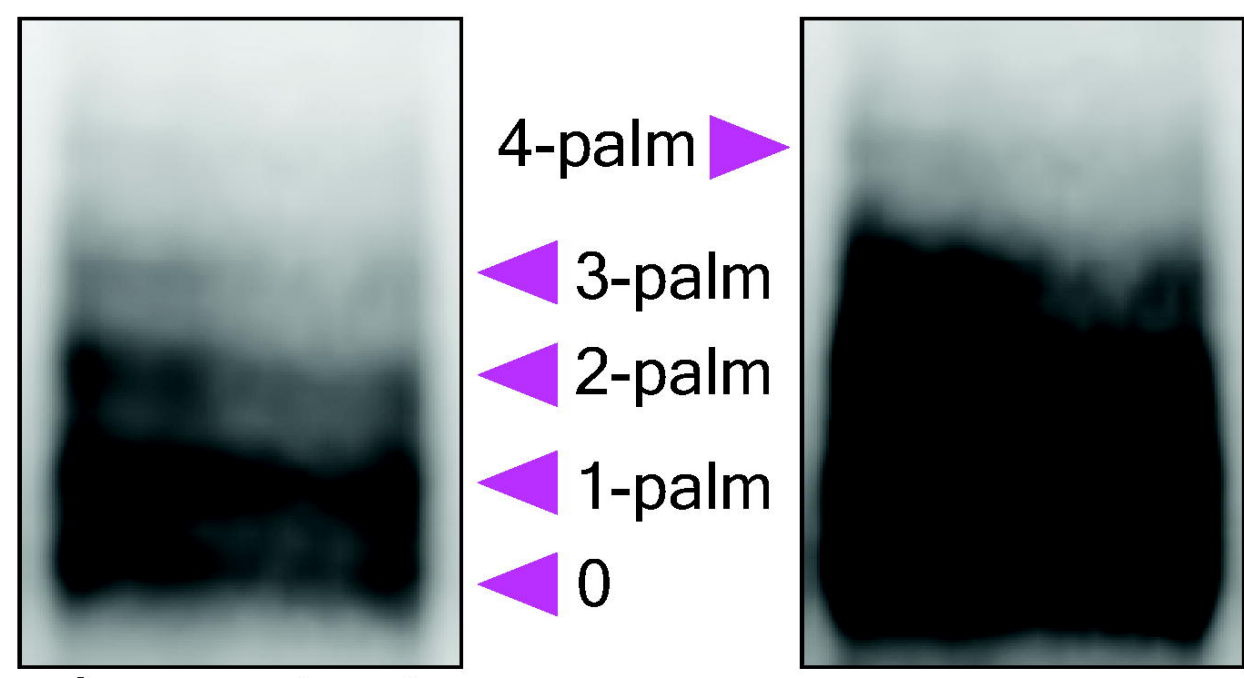

low contrast

high contrast 
bioRxiv preprint doi: https://doi.org/10.1101/2020.12.20.423603: this version posted December 21, 2020. The copyright holder for this preprint

Figure 2

A


C

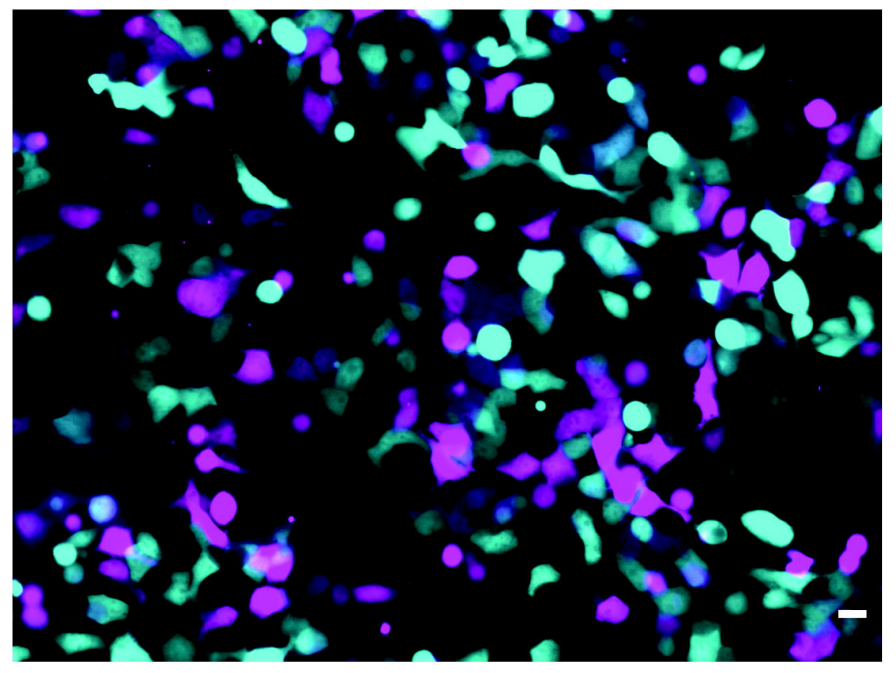

mCherry-C1

CoV-2 Spike-C9
B

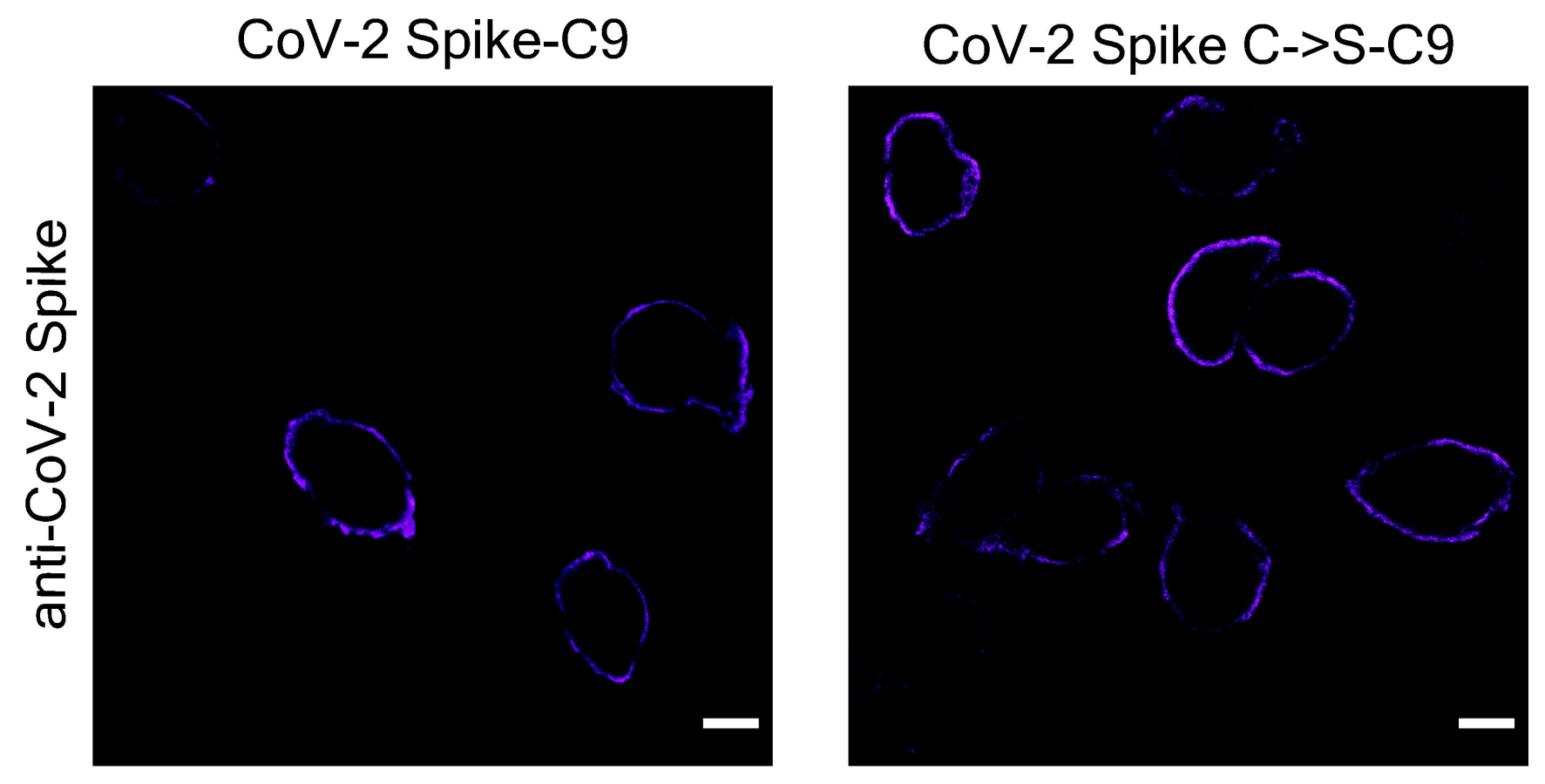

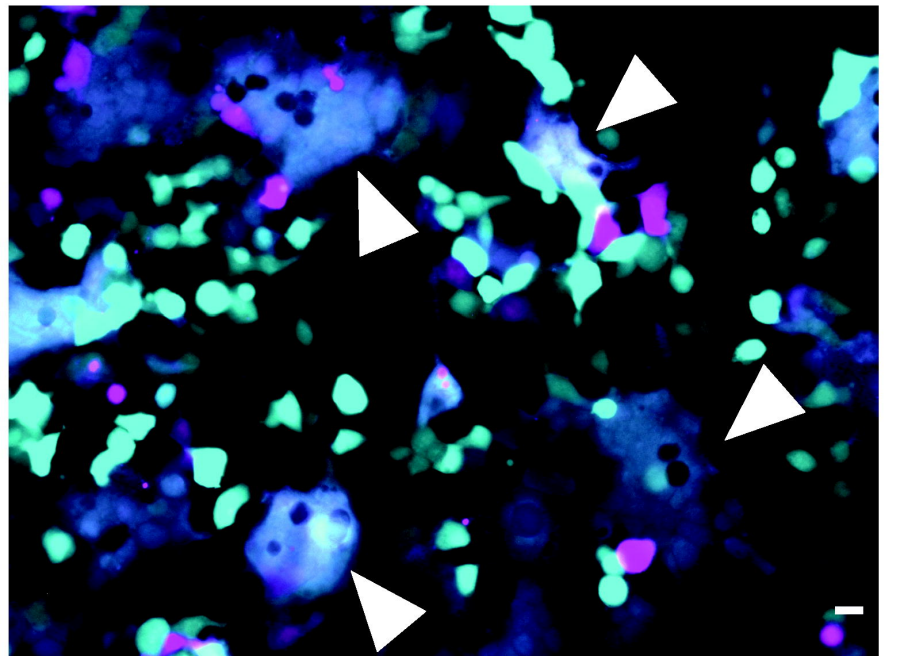

mCherry-C1 CoV-2 Spike-C9

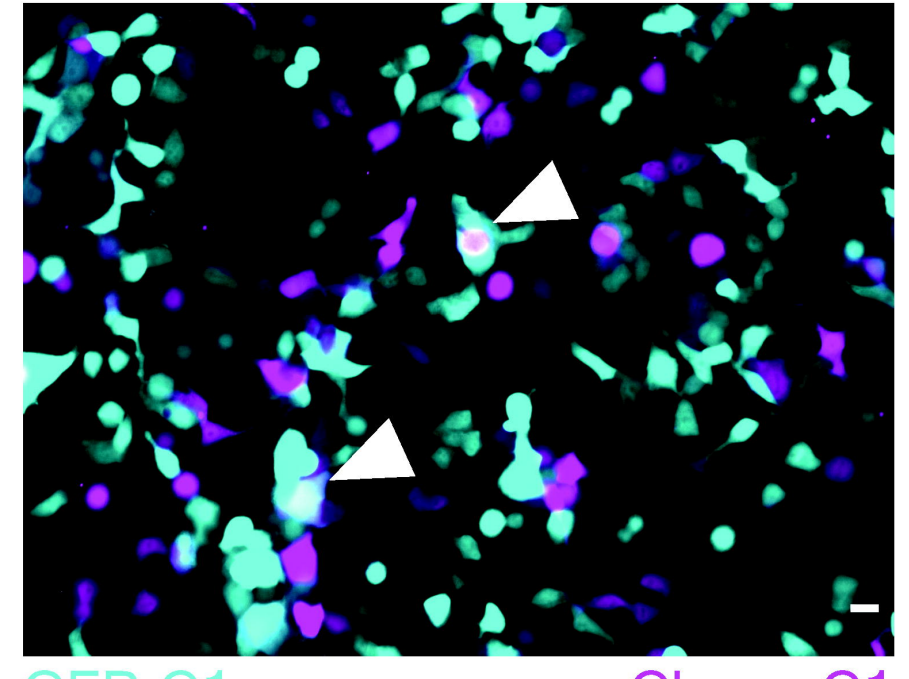

mCherry-C1

CoV-2 C->S Spike-C9 
bioRxiv preprint doi: https://doi.org/10.1101/2020.12.20.423603; this version posted December 21, 2020. The copyright holder for this preprint

Figurenen 3 .

A

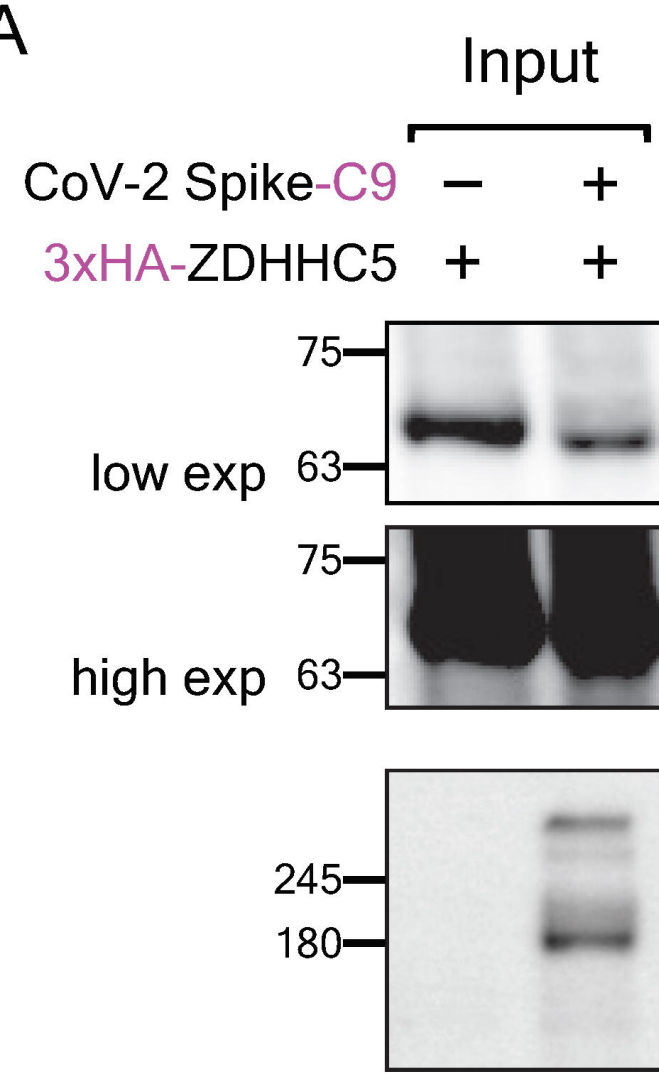

B

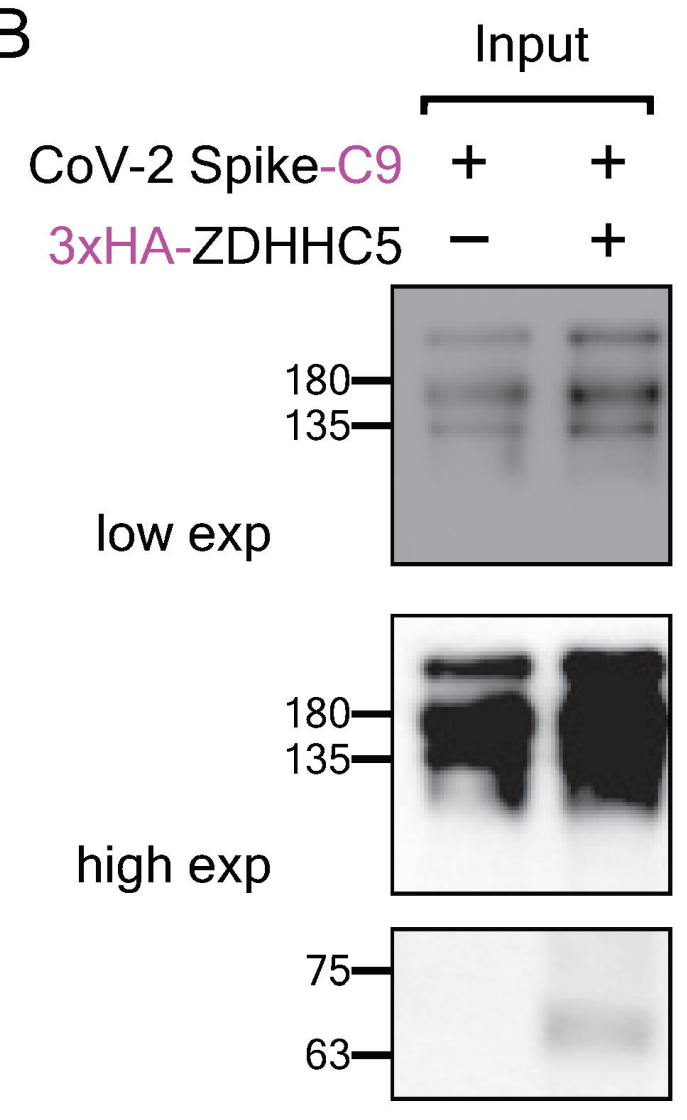

Co-IP C

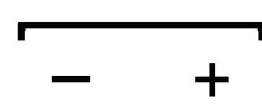

$+\quad+$

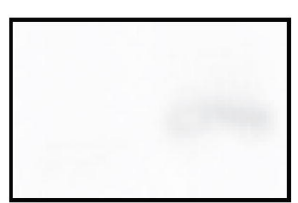

IP: C9

IB: HA


IB: C9
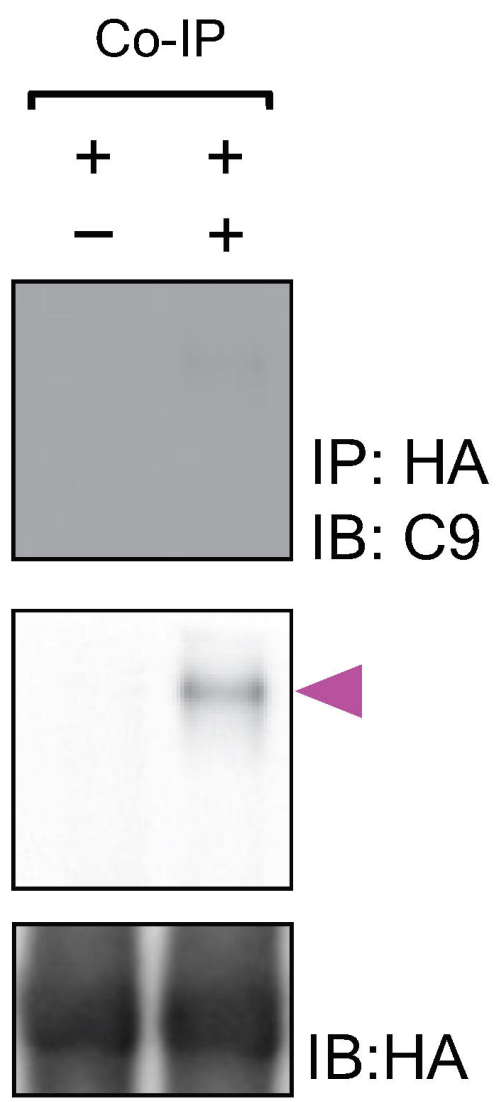

C

CoV-2Spike-C9 + + + + 3xHA-ZDHHC5 - - + +

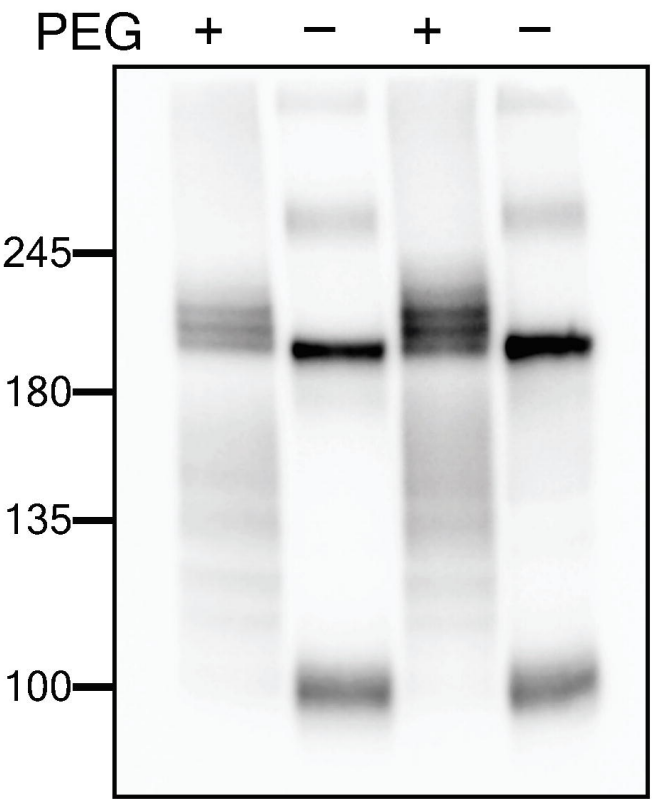

E

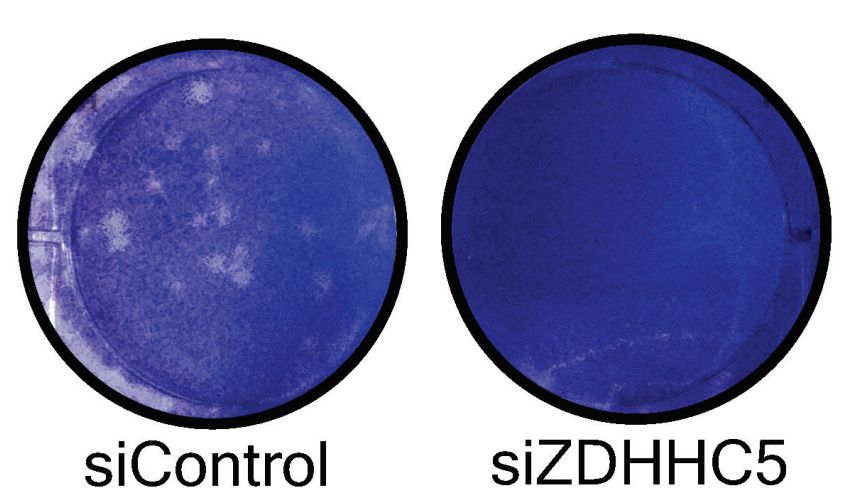

G

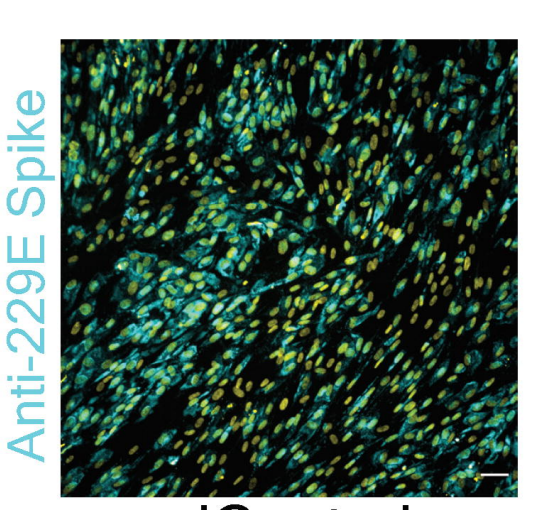

siControl



F

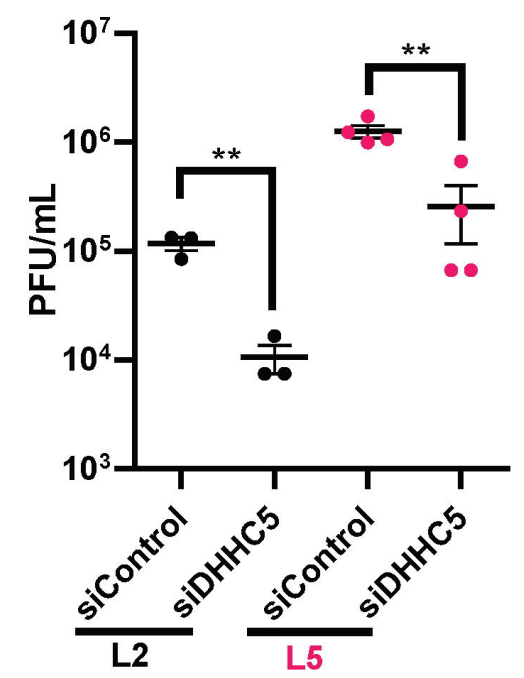

$\mathrm{H}$

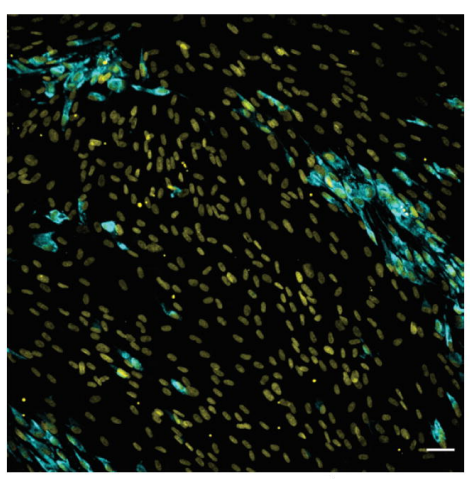

siZDHHC5




bioRxiv preprint doi: https://doi.org/10.1101/2020.12.20.423603; this version posted December 21 , 2020. The copyright holder for this preprint Figure 4 .

A

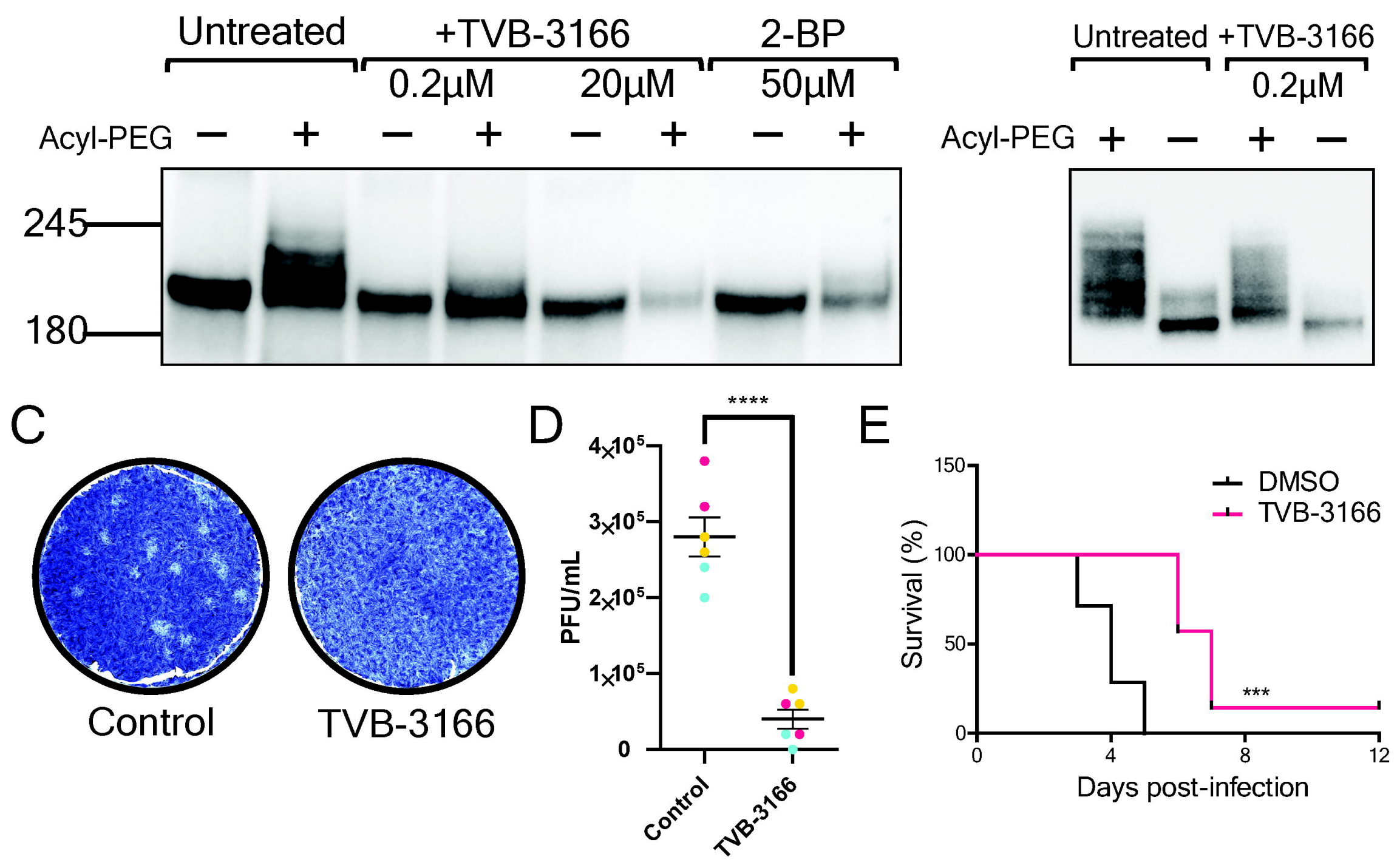

